\title{
Characterization of Micronized/Nanoscale Copper Particles in Wood Dust by TEM/ STEM
}

Chen Wang ${ }^{1}$, Chaolong Qi ${ }^{2}$, Alan S. Echt ${ }^{2}$, Alan K. Dozier ${ }^{1}$, Joseph E. Fernback $^{1}$, and M. Eileen Birch ${ }^{1}$

1. Division of Applied Research and Technology, National Institute for Occupational Safety and Health, Cincinnati, OH, 45226, USA

2. Division of Surveillance, Hazard Evaluations, and Field Studies, National Institute for Occupational Safety and Health, Cincinnati, OH, 45226, USA

In many wood preservative formulations, copper-based components are usually used as primary biocides to protect wood structures against microbial, fungal, and insect decay [1]. The wood preservation industry has recently introduced a particulate copper (i.e., micronized copper or MC) system as a replacement for ionized copper preservative in pressure-treated lumber (PTL) [2]. The use of MC treated wood in workplaces has raised concerns over the potential exposure of workers to the wood dust that contains micronized/nanoscale copper particles [3-4]. However, there is a lack of information about identification and characterization of copper particles in wood dust generated by the processing of MC treated PTL.

A laboratory testing system was used to generate wood dust to simulate the cutting of wood at construction sites. A Thermophoretic Personal Sampler (TPS100) was used to collect dust samples on a 300 mesh carbon-coated nickel TEM grid. The TEM samples were analyzed with a JEOL 2100F TEM equipped with an Energy Dispersive x-ray Spectroscopy (EDS) detector. Bright Field (BF) and High Angle Annular Dark Field (HAADF) images were acquired in the Scanning Transmission Electron Microscopy (STEM) mode with a probe size of 0.5-1 nm. For the regions of interest (ROI), selected point analysis and line scans were performed to collect the EDS spectra for elemental identification. Three major elements in the dust samples (carbon, copper and oxygen) were identified by their characteristic K lines. In addition, STEM spectrum images (STEM-SI) and Energy Filtered TEM (EFTEM) were used to analyze the elemental distributions at selected areas.

Results of the analyses of wood dust generated by cutting MC treated PTL are shown in Figs. 1 and 2. The presence of micronized/nanoscale copper particles was confirmed by STEM combined with EDS analysis. The copper agglomerates are embedded in the wood dust with sizes in the range from 5 to 1000 $\mathrm{nm}$. The composition analysis of individual nanoscale copper particles indicates the possible presence of copper carbonate. Samples generated under different testing conditions (i.e., test chamber flow rate and the type of saw blade) were analyzed. The results show that the size distributions of particles are nearly unchanged by different testing conditions examined.

\section{References:}

[1] M. H. Freeman and C.R. Mcintyre, Forest Prod. J., 58 (2008), 6-27.

[2] S.T. Lebow in "Wood Handbook - Wood as an engineering material" (Forest Service, US

Department of Agriculture, Washington, DC), 2010, 15-1.

[3] C. Civardi, F. W.M.R. Schwarze and P. Wick, Environ. Pollut., 200 (2015), 126-132.

[4] W.E. Platten et al., Sci. Total Environ., 548 (2016), 441-449. 

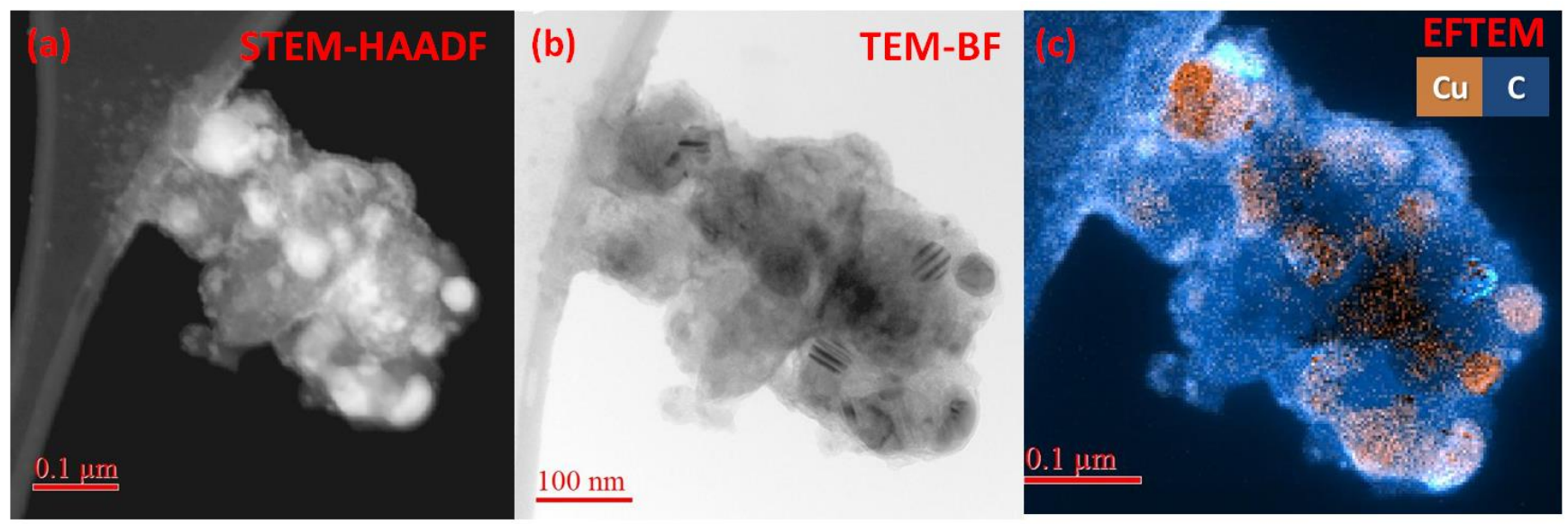

Figure 1. Images of wood dust with embedded nanoscale copper particles analyzed by (a) STEMHAADF; (b) TEM-BF; and (c) EFTEM.
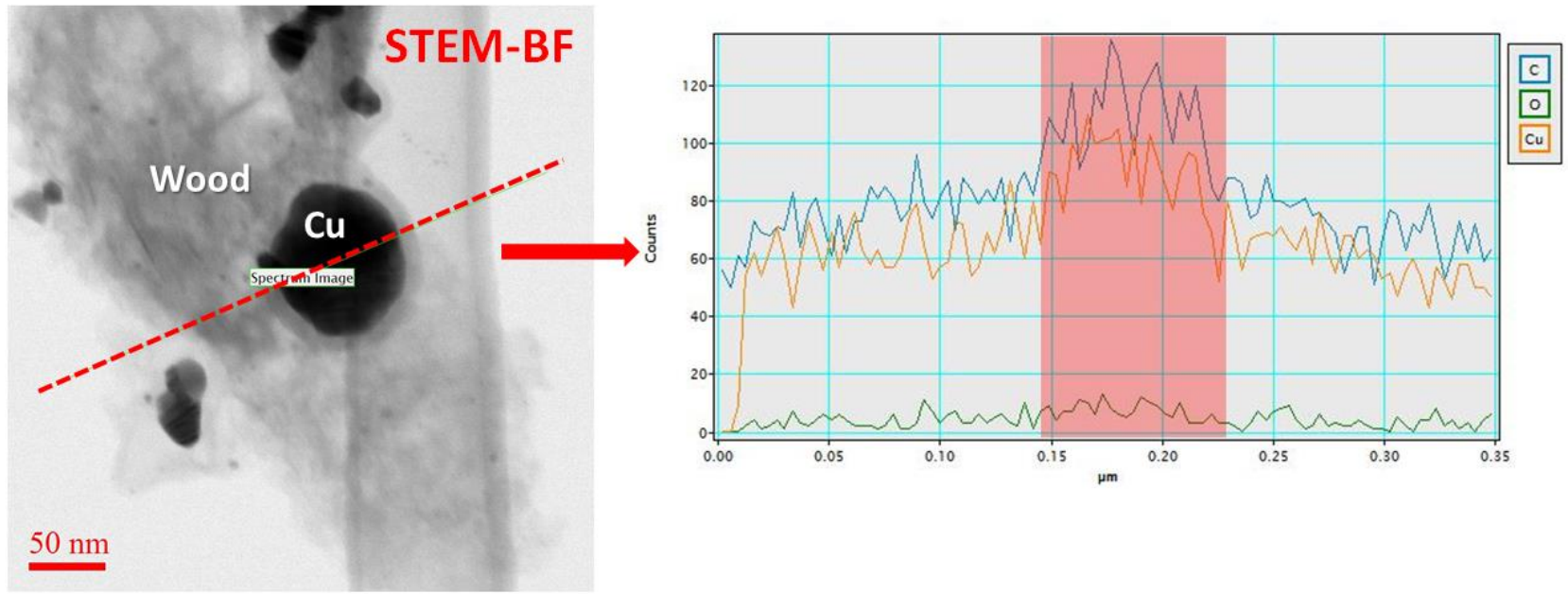

Figure 2. A line scan across a nanoscale particle was performed to confirm the presence of copper in the MC treated wood dust. 